Original article

\title{
Etch-and-rinse vs self-etch mode for dentin bonding effectiveness of universal adhesives
}

\author{
Kabun Yamauchi $^{1)}$, Akimasa Tsujimoto ${ }^{1)}$, Carlos A. Jurado ${ }^{2,3)}$, Yusuke Shimatani ${ }^{1)}$, Yuko Nagura ${ }^{1)}$, Toshiki Takamizawa ${ }^{1)}$, \\ Wayne W. Barkmeier ${ }^{4}$, Mark A. Latta ${ }^{4}$, and Masashi Miyazaki ${ }^{1)}$
}

1) Department of Operative Dentistry, Nihon University School of Dentistry, Tokyo, Japan

Department of Restorative Dentistry, Oregon Health and Science University School of Dentistry, Portland, OR, USA

Department of Digital Dentistry, A.T. Still University Arizona School of Dentistry, Mesa, AZ, USA

${ }^{4)}$ Department of General Dentistry, Creighton University School of Dentistry, Omaha, NE, USA

\begin{abstract}
In this study, dentin bond fatigue resistance and interfacial science characteristics of universal adhesives through etch-and-rinse and self-etch modes were investigated. Resin composite was bonded to human dentin with four universal adhesives, namely, Adhese Universal, All-Bond Universal, G-Premio Bond, and Scotchbond Universal Adhesive. The initial bond strengths, bond fatigue strengths, and interfacial science characteristics of the universal adhesives with dentin through etch-and-rinse and self-etch modes were determined. Bond fatigue resistance (initial bond strength and bond fatigue strength) of universal adhesives in etch-and-rinse mode showed no significant difference in contrast to that in self-etch mode and was material-dependent regardless of the etching mode. Although phosphoric acid conditioning of dentin did not have a strong impact on the bond fatigue resistance, surface free energy and parameters of dentin were significantly decreased by etching and by application of universal adhesives regardless of etching mode. Changes in $\gamma_{\mathrm{S}}$ and $\gamma_{\mathrm{s}}{ }^{\mathrm{h}}$ for when universal adhesive was applied to etched and ground dentin were significantly different depending on the adhesive. The results suggest that bonding performance of universal adhesives was effective in both etching modes; however, bonding mechanisms may be different for each.
\end{abstract}

Keywords; light-curing of dental adhesives, dental debonding, dental etching, dental restoration failure, dental stress analysis

\section{Introduction}

Universal adhesives have become a trend in dentistry because they can be used in either etch-and-rinse or self-etch mode, thanks to their reduced number of application steps and flexibility[1]. With the expiration of the patent for the 10-MDP (10-methacryloyloxydecyl dihydrogen phosphate) from Kuraray Noritake Dental in 2003, manufacturers began exploring the usage of 10-MDP and other phosphoric acid esterfor novel adhesive formulations. Scotchbond Universal Adhesive (3M Oral Care, St. Paul, MN, USA) was the first universal adhesive introduced commercially in Japan in November of 2012. Later, universal adhesives which could be of use with resin luting cements [2], various substrates without surface treatment [3], reduced treatment times [4], or various surface moisture of enamel and dentin surfaces [5] were introduced in the market. Despite this exciting flexibility, some reports suggest that the bond durability of numerous universal adhesives is inferior to that of the two-step self-etch adhesives. With enamel, this has been seen in etch-and-rinse and self-etch modes [6,7] and dentin in self-etch mode [8]. Nevertheless, flexibility of universal adhesives has enjoyed increasingly popularity in the clinical setting [9], hence the need to further investigate better ways to utilize universal adhesives.

Over the past decade, a method designed to assess bond fatigue resistance in adhesives has been developed by a collaborative effort between the Nihon University School of Dentistry (NU, Tokyo, Japan) and Creighton University School of Dentistry (CU, Omaha, NE, USA). Development started with Erickson et al. $[10,11]$ and was refined by researchers from

Correspondence to Dr. Akimasa Tsujimoto, Department of Operative Dentistry, Nihon University School of Dentistry, 1-8-13 Kanda-Surugadai, Chiyoda-ku, Tokyo 101-8310, Japan

Fax: +81-3-3219-8347 E-mail: tsujimoto.akimasa@nihon-u.ac.jp

J-STAGE Advance Publication: October 21, 2019

doi.org/10.2334/josnusd.18-0433

DN/JST.JSTAGE/josnusd/18-0433
NU and CU (Barkmeier et al. [12], Takamizawa et al. [13], and Tsujimoto et al. [14]). The appropriate frequency [13,15], number of cycles [14], and analysis are now well-established. However, at the present time, research comparing the bonding performance of universal adhesives using this bond fatigue test for either etch-and-rinse or self-etch mode has been limited.

Tian et al. [16] reported that the chemical interaction of 10-MDP with dentin is vital in obtaining durable bonds. Inoue et al. [17] showed that drawing from an interfacial science perspective, chemical bond interactions between 10-MDP and dentin could be explained through changes in dentin surface characteristics. Although it has been shown in the past that universal adhesives have similar bonding performance regardless of the bonding strategies employed, findings on the chemical bonding interactions of universal adhesive to ground and etched dentin may have been disparate. In addition, further assessment on the changes of energetic characteristics of dentin surfaces treated by universal adhesives in the two different modes, when added to the bond fatigue resistance analysis, may provide an explanation for the discrepancy between laboratory and clinical results.

The aim of this laboratory study was to assess dentin bond fatigue resistance and interfacial science characteristics of universal adhesives in etch-and-rinse and self-etch modes. The two null hypotheses tested were as follows: (i) etching mode would make no differences to the bond fatigue resistance of universal adhesives to dentin, and (ii) there would be no differences in the interfacial science characteristics of universal adhesivetreated dentin.

\section{Materials and Methods}

\section{Study materials}

Four universal adhesives were used in this study: (i) Adhese Universal (AU, Ivoclar Vivadent, Schaan, Liechtenstein), (ii) All-Bond Universal (AB, Bisco, Schaumburg, IL, USA), (iii) G-Premio Bond (GB, GC, Tokyo, Japan), and (iv) Scotchbond Universal Adhesive (SU, 3M Oral Care). Ultra-Etch (Ultradent, South Jordan, UT, USA) was used as a 35\% phosphoric acid pre-etching agent and Z100 Restorative (3M Oral Care) as the resin composite employed to make specimens. Table 1 lists the tested materials and their lot numbers and components.

\section{Specimen preparation}

This study used de-identified extracted human molar teeth. To comply with research ethics, the protocol was reviewed and approved by the Ethics Committee for Human Studies of NU (\#2015-06) and Biomedical Institutional Review Board at CU (\#760765-1). Preparation of the teeth was done in the manner described in the study by Nagura et al. [18], except that a flat dentin surface, rather than enamel surface, was prepared. Specimens with and without etching were prepared for each adhesive, giving a total of eight experimental groups for each of the procedures performed (Table 2).

\section{Initial bond strength testing}

Initial bond strength testing was carried out as described by Nagura et al. [18].

\section{Bond fatigue strength testing}

Bond fatigue strength testing was carried out as described by Nagura et al. [18]. 
Table 1 Materials used in this study

\begin{tabular}{|c|c|c|c|}
\hline Material & Type of material (Code) & Main components $(\mathrm{pH})$ & Manufacturer \\
\hline Adhese Universal (164453) & Universal adhesive (AU) & $\begin{array}{l}\text { Bis-GMA, HEMA, MDP, MCAP, decandiol dimethacrylate, dimethacrylate, ethanol, } \\
\text { water, initiator, stabilizers, silicon dioxide ( } 2.5)\end{array}$ & $\begin{array}{l}\text { Ivoclar Vivadent, Schaan, } \\
\text { Liechtenstein }\end{array}$ \\
\hline $\begin{array}{l}\text { All-Bond Universal } \\
(1300008503)\end{array}$ & Universal adhesive $(\mathrm{AB})$ & Bis-GMA, HEMA, MDP, ethanol, water, initiators (3.2) & Bisco, Schaumburg, IL, USA \\
\hline G-Premio Bond (1603091) & Universal adhesive (GB) & MDP, 4-MET, MEPS, methacrylate monomer, acetone, water, initiator, silica (1.5) & GC, Tokyo, Japan \\
\hline $\begin{array}{l}\text { Scotchbond Universal Adhesive } \\
\text { (617265) }\end{array}$ & Universal adhesive (SU) & $\begin{array}{l}\text { Bis-GMA, HEMA, decamethylene dimethacrylate, ethyl methacryalate, propenoic } \\
\text { acid, methyl-reaction products with decanediol and phosphorous oxide, copolymer of } \\
\text { acrylic and itaconic acid, dimethylaminobenzoate, methyl ethyl ketone, ethanol, water, } \\
\text { silane-treated silica, initiator }(2.7)\end{array}$ & $\begin{array}{l}\text { 3M Oral Care, } \\
\text { St. Paul, MN, USA }\end{array}$ \\
\hline Ultra-Etch (G019) & Pre-etching agent & $35 \%$ phosphoric acid, glycol, cobalt aluminate blue spinel & $\begin{array}{l}\text { Ultradent Products, South } \\
\text { Jordan, UT, USA }\end{array}$ \\
\hline Z100 (1312131) & Resin composite (Z100) & Bis-GMA, TEGDMA, silane-treated ceramic, benzotriazolyl methylphenol & 3M ESPE \\
\hline
\end{tabular}

acid polymer; 4-MET, 4-methacryloyloxyethyl trimellitate; MEPS, methacryloyloxyalkyl thiophosphate methylmethacrylate; TEGDMA, triethylene glycol dimethacrylate

Table 2 Application protocols used for phosphoric acid etching and for adhesive application

\begin{tabular}{ll}
\hline Etching mode & Phosphoric acid pre-etching protocol \\
\hline $\begin{array}{l}\text { Etch-and-rinse mode } \\
\text { Self-etch mode }\end{array}$ & Dentin surface was conditioned with phosphoric acid for $15 \mathrm{~s}$. Conditioned surface was rinsed with water for $15 \mathrm{~s}$ (three-way dental syringe) and air-dried \\
\hline Adhesive & Phosphoric acid etching was not performed \\
\hline AU & Adhesive application protocol \\
AB & Adhesive applied to air-dried tooth surface with rubbing action for $20 \mathrm{~s}$ and then medium air pressure applied to surface for $5 \mathrm{~s}$. Photo-polymerized for $10 \mathrm{~s}$ \\
GB & Adhesive applied to enamel surface with rubbing action for $10-15 \mathrm{~s}$ and then medium air pressure applied to surface for $10 \mathrm{~s}$. Photo-cured for $10 \mathrm{~s}$ \\
SU & Adhesive applied to air-dried tooth surface for $10 \mathrm{~s}$ and then maximum air pressure applied to surface for $5 \mathrm{~s}$. Photo-cured for $10 \mathrm{~s}$ \\
& Adhesive applied to air-dried tooth surface with rubbing action for $20 \mathrm{~s}$ and then medium air pressure applied to surface for $5 \mathrm{~s}$. Photo-cured for $10 \mathrm{~s}$ \\
\hline
\end{tabular}

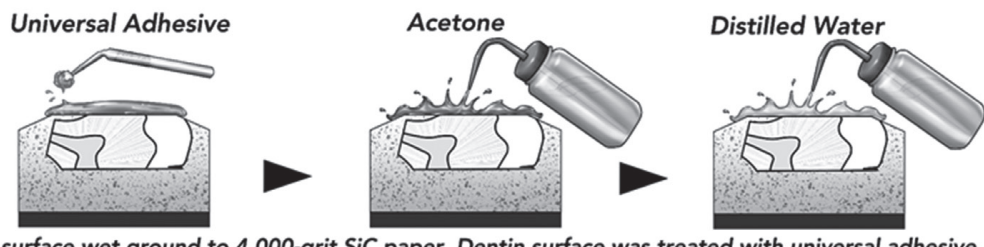

Dentin surface wet ground to 4,000-grit SiC paper. Dentin surface was treated with universal adhesive, and the uncured adhesive layer was removed using acetone and distilled water

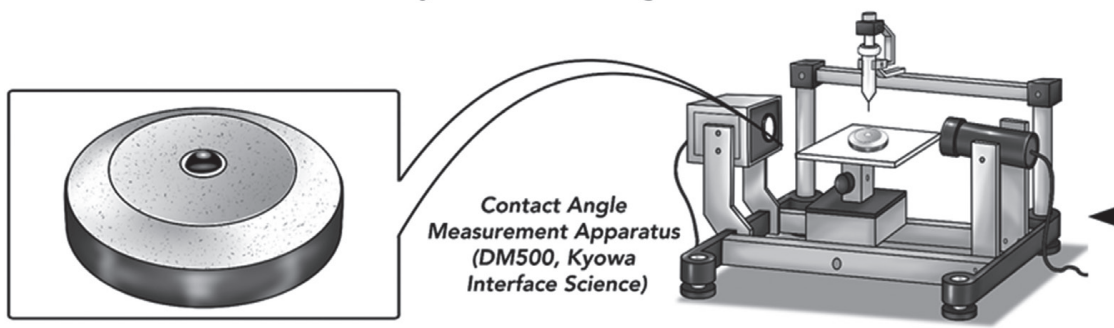

Contact angle of specimen was measured using bromonaphthalene, diiodomethane and distilled water

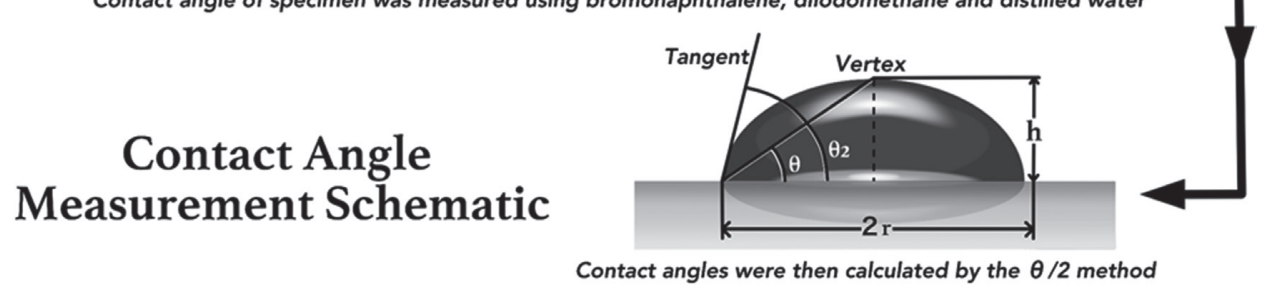

Fig. 1 Illustration of the apparatus for contact angle measurement

Failure mode of specimens

Failure mode was assessed as described by Nagura et al. [18].

Interfacial science characteristics measurement

Interfacial science characteristics were measured as described by Nagura et al. [18]. The procedure is depicted in Fig. 1.

\section{Scanning electron microscopy (SEM) observation of bonding} interface

SEM observations were conducted as described by Nagura et al. [18].

\section{Statistical analysis}

Statistical analysis was conducted as described by Nagura et al. [18], with the exception that Fisher's exact test was not used in this study.

\section{Results}

\section{Initial bond strength}

In Table 3 are the initial bond strengths of the universal adhesives in both etching mode groups. The initial bond strengths showed no influence $(P>$ 0.05 ) by the etching mode, and the percentage difference of the initial bond strength between the two modes was within 10\% (3-10\%). The initial bond strength was material-dependent regardless of etching mode.

\section{Bond fatigue strength}

Table 4 shows the bond fatigue strengths in both etching mode groups. 
Table 3 Initial shear bond strength of universal adhesives to dentin using etch-and-rinse and self-etch modes

\begin{tabular}{lccc}
\hline Adhesive & Etch-and-rinse mode & Self-etch mode & Percentage difference \\
\hline $\mathrm{AU}$ & $29.0(4.2)^{\mathrm{a}, \mathrm{A}}$ & $26.4(5.5)^{\mathrm{a}, \mathrm{A}}$ & $10 \%$ \\
$\mathrm{AB}$ & $27.2(3.8)^{\mathrm{a}, \mathrm{A}}$ & $27.9(3.7)^{\mathrm{a}, \mathrm{A}}$ & $3 \%$ \\
$\mathrm{~GB}$ & $26.2(5.5)^{\mathrm{a}, \mathrm{A}}$ & $27.5(3.5)^{\mathrm{a}, \mathrm{A}}$ & $5 \%$ \\
$\mathrm{SU}$ & $39.3(4.6)^{\mathrm{b}, \mathrm{A}}$ & $41.5(4.5)^{\mathrm{b}, \mathrm{A}}$ & $6 \%$ \\
\hline
\end{tabular}

Unit: MPa. Values in parenthesis are standard deviations. Same small letters in same individual column indicate no significant difference $(P>0.05)$. Same capital letters within individual rows indicate no significant difference $(P>0.05)$

Table 4 Bond fatigue strength of universal adhesives to dentin using etch-and-rinse and self-etch modes

\begin{tabular}{lccc}
\hline Adhesive & Etch-and-rinse mode & Self-etch mode & Percentage difference \\
\hline AU & $13.7(1.9)^{\mathrm{a}, \mathrm{A}}$ & $12.3(3.3)^{\mathrm{a}, \mathrm{A}}$ & $11 \%$ \\
$\mathrm{AB}$ & $13.1(2.5)^{\mathrm{a}, \mathrm{A}}$ & $13.8(2.4)^{\mathrm{a}, \mathrm{A}}$ & $5 \%$ \\
$\mathrm{~GB}$ & $13.0(2.2)^{\mathrm{a}, \mathrm{A}}$ & $14.3(2.8)^{\mathrm{a}, \mathrm{A}}$ & $10 \%$ \\
$\mathrm{SU}$ & $19.0(3.1)^{\mathrm{b}, \mathrm{A}}$ & $20.6(2.8)^{\mathrm{b}, \mathrm{A}}$ & $8 \%$ \\
\hline
\end{tabular}

Unit: MPa. Values in parenthesis are standard deviations. Same small letters in same individual column indicate no significant difference $(P>0.05)$. Same capital letters within individual rows indicate no significant difference $(P>0.05)$
Table 5 Failure mode analysis of debonded specimens after initial bond strength testss

\begin{tabular}{lcc}
\hline Adhesive & Etch-and-rinse mode & Self-etch mode \\
\hline $\mathrm{AU}$ & {$[86 / 0 / 7 / 7]$} & {$[100 / 0 / 0 / 0]$} \\
$\mathrm{AB}$ & {$[100 / 0 / 0 / 0]$} & {$[100 / 0 / 0 / 0]$} \\
$\mathrm{GB}$ & {$[86 / 0 / 7 / 7]$} & {$[100 / 0 / 0 / 0]$} \\
$\mathrm{SU}$ & {$[86 / 0 / 14 / 0]$} & {$[100 / 0 / 0 / 0]$} \\
\hline
\end{tabular}

Percentage of failure mode [adhesive failure/cohesive failure in resin/cohesive failure in enamel/ mixed failure]. Same small letters in same column indicate no significant difference $(P>0.05)$. Same capital letters within individual rows indicate no significant difference $(P>0.05)$.

Table 6 Failure mode analysis of debonded specimens after shear fatigue strength test

\begin{tabular}{lcc}
\hline Adhesive & Etch-and-rinse mode & Self-etch mode \\
\hline $\mathrm{AU}$ & {$[90 / 0 / 5 / 5]$} & {$[100 / 0 / 0 / 0]$} \\
$\mathrm{AB}$ & {$[90 / 0 / 10 / 0]$} & {$[100 / 0 / 0 / 0]$} \\
$\mathrm{GB}$ & {$[80 / 0 / 10 / 10]^{\mathrm{a}}$} & {$[90 / 0 / 10 / 0]$} \\
$\mathrm{SU}$ & {$[60 / 10 / 20 / 10]$} & {$[100 / 0 / 0 / 0]$} \\
\hline
\end{tabular}

Percentage of failure mode [adhesive failure/cohesive failure in resin/cohesive failure in enamel/ mixed failure]. Same small letters in same column indicate no significant difference $(P>0.05)$. Same capital letters within individual rows indicate no significant difference $(P>0.05)$.

Table 7 Surface free energy characteristics of universal adhesive-treated dentin using total-etch and self-etch modes

\begin{tabular}{|c|c|c|c|c|c|c|c|c|}
\hline \multirow{2}{*}{ Adhesive } & \multicolumn{4}{|c|}{ Etch-and-rinse mode } & \multicolumn{4}{|c|}{ Self-etch mode } \\
\hline & $\gamma \mathrm{s}$ & $\gamma \mathrm{s}^{\mathrm{d}}$ & $\gamma \mathrm{s}^{\mathrm{p}}$ & $\gamma \mathrm{s}^{\mathrm{h}}$ & $\gamma \mathrm{s}$ & $\gamma \mathrm{s}^{\mathrm{d}}$ & $\gamma \mathrm{s}^{\mathrm{p}}$ & $\gamma \mathrm{s}^{\mathrm{h}}$ \\
\hline baseline & $41.1(2.5)^{\mathrm{a}}$ & $37.8(1.5)^{\mathrm{a}}$ & $1.1(1.6)^{\mathrm{a}}$ & $2.2(1.1)^{\mathrm{a}}$ & $68.8(3.6)^{\mathrm{a}}$ & $41.0(1.4)^{\mathrm{a}}$ & $2.8(2.3)^{\mathrm{a}}$ & $25.0(2.4)^{\mathrm{a}}$ \\
\hline $\mathrm{AU}$ & $39.6(2.3)^{\mathrm{a}, \mathrm{b}}$ & $37.0(1.7)^{\mathrm{a}}$ & $1.4(2.1)^{\mathrm{a}}$ & $1.2(0.9)^{\mathrm{b}}$ & $65.5(3.7)^{\mathrm{b}}$ & $40.0(1.4)^{\mathrm{a}}$ & $2.6(2.2)^{\mathrm{a}}$ & $22.9(2.6)^{\mathrm{b}}$ \\
\hline $\mathrm{AB}$ & $39.0(2.2)^{\mathrm{a}, \mathrm{b}}$ & $37.1(0.4)^{\mathrm{a}}$ & $1.0(1.9)^{\mathrm{a}}$ & $0.9(2.0)^{\mathrm{b}}$ & $63.8(3.0)^{\mathrm{b}}$ & $40.1(0.4)^{\mathrm{a}}$ & $2.4(1.7)^{\mathrm{a}}$ & $21.3(2.2)^{\mathrm{b}}$ \\
\hline GB & $37.7(1.9)^{\mathrm{b}}$ & $37.2(1.6)^{\mathrm{a}}$ & $0.4(1.0)^{\mathrm{a}}$ & $0.1(0.3)^{\mathrm{c}}$ & $61.1(3.0)^{\mathrm{b}, \mathrm{c}}$ & $40.3(1.5)^{\mathrm{a}}$ & $2.6(2.1)^{\mathrm{a}}$ & $18.2(3.1)^{b, c}$ \\
\hline SU & $38.0(2.2)^{\mathrm{b}}$ & $37.1(1.6)^{\mathrm{a}}$ & $0.7(1.5)^{\mathrm{a}}$ & $0.2(0.4)^{\mathrm{d}}$ & $62.0(3.7)^{\mathrm{b}, \mathrm{c}}$ & $40.8(1.5)^{\mathrm{a}}$ & $1.5(2.0)^{\mathrm{a}}$ & $19.7(2.7)^{b, c}$ \\
\hline
\end{tabular}

Bond fatigue strengths were not influenced $(P>0.05)$ by the etching mode, and percentage differences of bond fatigue strength between the two modes were less than $11 \%(5-11 \%)$. The bond fatigue strength was material-dependent regardless of the etching mode.

\section{Failure mode analysis}

The failure mode analysis for initial bond strength is shown in Table 5, while that for bond fatigue strength testing is in Table 6. Most of the failure modes were adhesive failure, and neither type of adhesive nor the etching mode made a difference.

\section{Interfacial science characteristics of adhesive-treated dentin}

Table 7 shows the interfacial science characteristics of dentin treated with the universal adhesives. The baseline in the etch-and-rinse group exhibited a significantly lower $\gamma_{\mathrm{S}}$ and $\gamma_{\mathrm{S}}{ }^{\mathrm{h}}(P<0.05)$ than that in the self-etch group. Changes in $\gamma_{\mathrm{s}}{ }^{\mathrm{d}}$ and $\gamma_{\mathrm{s}}{ }^{\mathrm{p}}$ were found to have not been influenced $(P>0.05)$ by the type of adhesive used, in contrast to changes in $\gamma_{\mathrm{s}}{ }^{\mathrm{h}}$ which were observed to be influenced by type of adhesive $(P<0.05)$.

\section{SEM observation of bonding interface}

Figure 2 shows representative SEM images of resin-dentin interfaces from both etching groups. The adhesive layer was approximately 7-12 $\mu \mathrm{m}$ thick, and the adaptation of the interface to dentin was excellent for both etching modes. However, cracks were visible in the adhesive layer of the resindentin interface of GB in both groups, but not in the other adhesives.

\section{Discussion}

Research into the application of "compressive shear load" in a conventional shear bond strength test using finite element stress analysis was previously conducted by Van Noort et al. [19] and has shown lack of uniform distributed load along the bonding interface. This was also confirmed through the recent study reported by Jin et al. [20]. The conventional shear bond strength test has long been criticized as neither appropriate nor reliable for the measurement of "true" or "actual" shear bond strength at the bonding interface and may have been measuring "mixed" shear bond strength.
Especially in the modified shear bond strength test using dynamic loading in this study, a non-uniform stress distribution which is made by shear stress may be a debatable larger issue than in the conventional shear bond strength test due to the repeated stress loading. A mold-enclosed format was utilized in this study in which a stainless steel metal ring encloses the resin composite when both initial bond and fatigue strength are measured. It has been reported that with utilization of this method, tensile stress is significantly reduced while desired shear stress is maintained in shear bond strength tests, and finite element analysis suggests that it is more suitable for these measurements [20]. Aside from minimizing radius effects at the bonding interface, the mold-enclosed method has the ability to reduce load bearing on the resin composite itself, as the force is applied indirectly through the metal ring [21,22]. Barkmeier et al. [12] previously reported that with metal ring-enclosed method employed, shear bond strengths were generally $20 \%$ higher than the results achieved with use of Ultradent shear bond strength method. Hence, the mold-enclosed method was chosen as a fitting tool for bond fatigue resistance analysis of universal adhesives.

The results of the present study showed that etching mode did not affect the bond fatigue resistance of universal adhesives to dentin. However, the first null hypothesis that etching mode would make no difference to the bond fatigue resistance of universal adhesives to dentin was not rejected. A recent study [18], which uses the same research design to study bonding to enamel, has reported that the bond fatigue resistance of universal adhesives was significantly higher in etch-and-rinse than in self-etch mode. The results of the present and previous studies suggested that the use of etch-and-rinse or selective etching modes with universal adhesives is more effective from the bond fatigue resistance perspectives in laboratory, in agreement with the systematic review of earlier laboratory bond strength evaluations [23].

Results for the interfacial science characteristic evaluation show the baseline $\gamma_{\mathrm{S}}, \gamma_{\mathrm{S}}^{\mathrm{d}}, \gamma_{\mathrm{S}}{ }^{\mathrm{p}}$, and $\gamma_{\mathrm{s}}{ }^{\mathrm{h}}$ of dentin in the etch-and-rinse group (phosphoric acid etched dentin) were different and significantly below those in the self-etch group (ground dentin), where $\gamma_{\mathrm{s}}$ is the total surface free energy; $\gamma_{S}{ }^{d}$ is the dispersion force of the solid surface which is related to its wettability [17]; $\gamma \mathrm{s}^{\mathrm{p}}$ is the polarity force, which represents degree of polarization; and $\gamma \mathrm{s}^{\mathrm{h}}$ is the hydrogen-bonding force, which indicates 

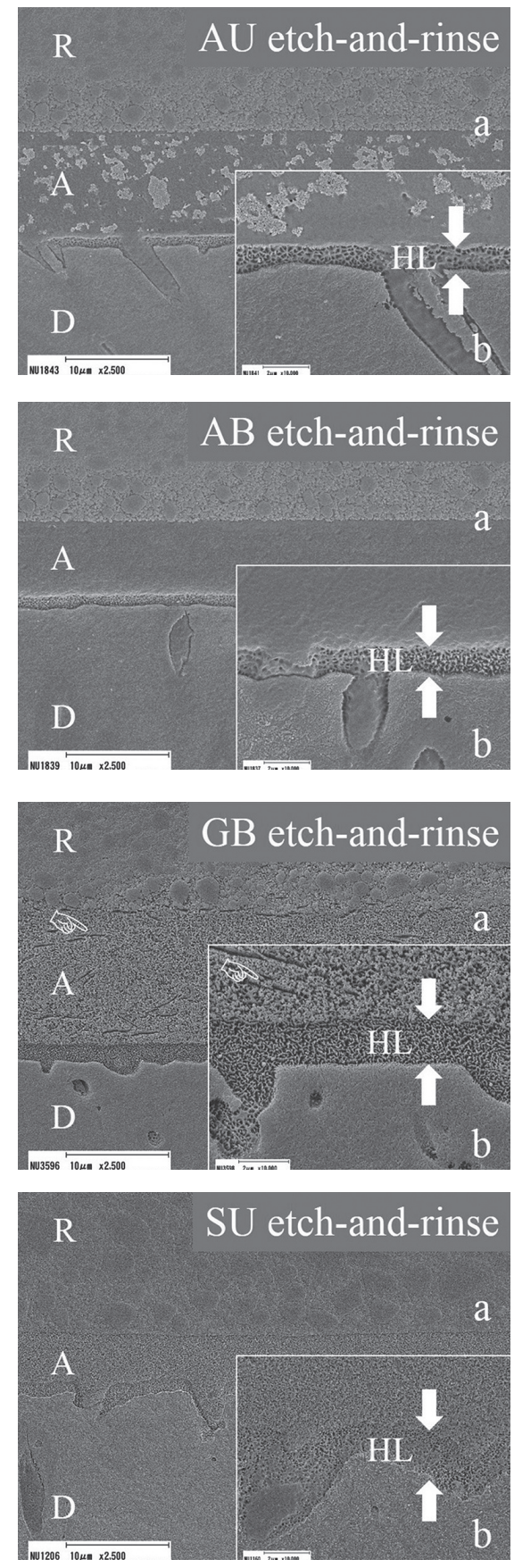
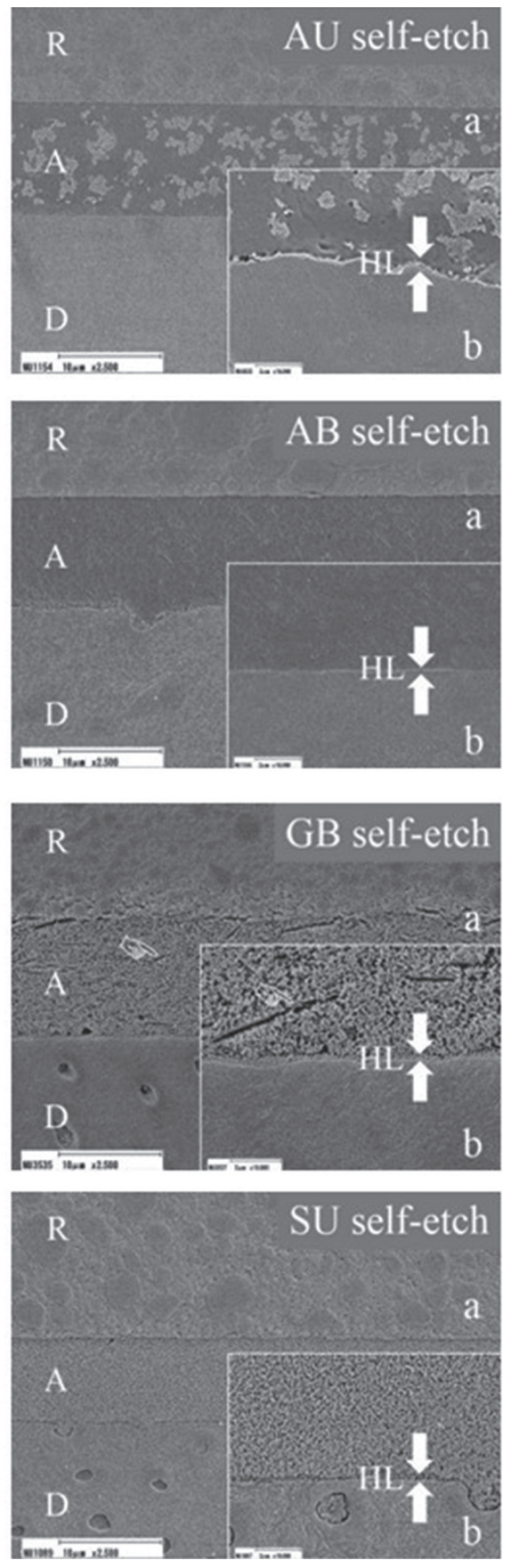

Fig. 2 Typical scanning electron microscopy images of the resin-dentin interface of etch-and-rinse and self-etch groups at (a) $2,500 \times$ magnification and (b) $10,000 \times$ magnification . A, Adhesive; D, dentin; HL, hybrid layer; R, resin composite; AU, Adhese Universal; AB, All-Bond Universal; GB, G-Premio Bond; SU, Scotchbond Universal Adhesive

the hydrophilicity of the surface [24]. This means that phosphoric acid etched dentin has lower wettability and degree of polarization and is less hydrophilic compared to ground dentin. Tay et al. $[25,26]$ have repeatedly reported that dehydration of demineralized dentin results in osmosis of water content from deeper dentin, leading to weaker bonding due to osmotic blisters and hydrolysis of the adhesive itself. However, bonding strategy did not influence the bond fatigue resistance of these adhesives to dentin. In the SEM observation of adhesive interfaces in etch-and-rinse mode, the deeper penetration of adhesives into the dentinal tubules was observed due to the removal of the smear layer and opening of the dentinal tubules by phosphoric acid etching, despite the lower wettability of the dentin. These deeper resin tags, which were perpendicular to shear stress, may have contributed to the higher resistance of adhesive interfaces to cyclic fatigue. In addition, fatigue testing was done with $20 \mathrm{~Hz}$ frequency and a cycling period of 50,000 cycles, in approximately 40 minutes, which is not very long. Hence, influence of osmosis of water content from deeper dentin, resulting in osmotic blisters and hydrolysis of adhesive, might be less due to the shorter period of testing compared to tests using long-term water storage or thermal cycling.

However, the bond fatigue resistance of universal adhesives showed a dependence on the material. The bond fatigue resistance of SU was significantly higher than the other adhesives, regardless of etching mode. Although no clear relationship between bond fatigue resistance and interfacial science characteristics was seen, the measurements of interfacial science characteristics of adhesive-treated dentin may make differences among the adhesives tested clearer. For universal adhesives, 10-MDP is a key technological factor for chemical bonding with tooth substrates, regardless of the bonding strategies employed [16]. The adhesion-decalcification concept [27] claims that the functional group of 10-MDP interacts ionically with calcium in dentin and forms a chemically bonded 10-MDP-calcium salt-layered structure on the dentin surface. As the long carbonyl chain of 10-MDP is relatively hydrophobic [28], a hydrophobic layer would cover the dentin surface due to the 10-MDP-calcium salt-layered structure. A greater extent of chemical bonding to the dentin surface should therefore create a more hydrophobic surface. The results for $\gamma_{\mathrm{S}}$ and $\gamma_{\mathrm{s}}{ }^{\mathrm{h}}$ on universal adhesive-treated dentin surfaces depended on the material regardless of 
etching mode, unlike $\gamma_{\mathrm{s}}{ }^{\mathrm{d}}$ and $\gamma_{\mathrm{s}}{ }^{\mathrm{p}}$. Thus, the second null hypothesis that there would be no differences in the interfacial science characteristics of universal adhesive-treated dentin was rejected. Further, $\gamma_{\mathrm{S}}$ and $\gamma_{\mathrm{S}}{ }^{\mathrm{h}}$ on GB- and SU-treated surfaces in both etching groups were significantly lower than those of AU and AB. Previous studies [16,23,24] reported that interfacial science characteristics were influenced by the reaction between acidic functional monomers and calcium in tooth substrates; thus these results were consistent with previous studies. Furthermore, the interfacial science characteristics of adhesive-treated surfaces for all tested adhesives decreased compared to those of the baseline regardless of etching mode, suggesting that the adhesives were capable of forming chemical bonds to both etched and unetched dentin, creating a surface layer with distinct chemical properties.

On the other hand, even though GB-treated dentin had a lower $\gamma \mathrm{s}$, similar to SU, GB showed a lesser bond fatigue durability than SU, and there was no significant difference from $\mathrm{AU}$ or $\mathrm{AB}$. In SEM, observations revealed clear qualitative differences in the adhesive layers. Cracks and defects were observed in the adhesive layer of GB-dentin interfaces, in both etching modes, but not in the other adhesives tested. A previous study of the water content of universal adhesives [29] reported that GB contains approximately $25 \%$ water, a higher proportion than other universal adhesives, where the proportion can be as low as $3 \%$ (in AU). SU has approximately half the proportion of water found in GB (i.e., 10 to $15 \%$ ). The extra water in GB is present to enhance its demineralization of tooth substrates. The higher level of water content in GB might lead to weakness caused by cracks, especially residual water in the cured adhesive layer. This partially explains why the bond fatigue resistance of GB was lower than that of SU and similar to the other adhesives, even though GB-treated dentin showed lower surface free energies.

GB also has a distinctive composition: The three other adhesives used in the study contained a single functional monomer, 10-MDP, while GB on the other hand contains several acidic functional monomers, which include 10-MDP, 4-methacryloxyethyl trimellitic acid (4-MET), and 10-methacryloyloxydecyl dihydrogen thiophosphate (10-MDTP). The bonding mechanisms of 4-MET and 10-MDP to dentin have been reported to be similar [30], creating a hydrophobic surface. Given this, it is reasonable to suppose that multiple monomers might contribute to lower $\gamma_{\mathrm{S}}$ and $\gamma_{\mathrm{S}}{ }^{\mathrm{h}}$ values of GB-treated dentin. Conversely, a previous study has reported that 4-MET has substantially lower chemical bonding potential than 10-MDP [31]. If 4-MET makes a substantial contribution to reducing $\gamma_{\mathrm{S}}$ and $\gamma_{\mathrm{S}}{ }_{\mathrm{h}}^{\mathrm{h}}$, but a smaller contribution to bond durability, this may also help to explain why GB had a lower bond fatigue resistance than SU despite the similar interfacial science characteristics results.

Taken together, these results suggest that the bonding mechanism in universal adhesives may vary substantially between adhesives and between application modes for the same adhesive. A better understanding of these mechanisms may allow significant improvements to be made to the bonding performance of universal adhesives, and is thus an important topic for further research.

Considering the results of present and previous studies which assessed enamel and dentin bond fatigue resistance with universal adhesives, universal adhesives used in etch-and-rinse mode may have better bond fatigue resistance because the etch-and-rinse mode leads to higher enamel bonding than self-etch mode and does not make a significant difference to dentin bond fatigue resistance. However, clinical studies [6,7] found there was no statistical difference among bonding strategies when a universal adhesive was used and that etching had only a minor effect with universal adhesives. These results may indicate that the observed increase of enamel bond fatigue resistance in etch-and-rinse mode fails to make an important clinical difference, most likely because dentin bond fatigue resistance has a much stronger influence. Research to clarify the relationship between bond fatigue resistance and clinical results would make a valuable contribution to the field.

\section{Acknowledgments}

The authors would like to thank Mr. Jason M. Moody for his technical contribution to this study.

\section{Conflict of Interest}

The authors of this manuscript certify that they have no proprietary, finan- cial, or other personal interest of any nature or kind in any product, service, and/or company that is presented in this article.

\section{References}

1. Jang JH, Lee MG, Woo SU, Lee CO, Yi JK, Kim DS (2016) Comparative study of the dentin bond strength of a new universal adhesive. Dent Mater J 35, 606-612.

2. Tsujimoto A, Barkmeier WW, Takamizawa T, Watanabe H, Johnson WW, Latta MA et al. Simulated localized wear of resin luting cement for universal adhesive systems with different curing mode. J Oral Sci 2018, 60, 29-36.

3. Tsujimoto A, Barkmeier WW, Takamizawa T, Wilwerding T, Latta MA, Miyazaki M (2017) Interfacial characteristics and bond durability on universal adhesives to various substrates. Oper Dent 42, e59-e70.

4. Nagura Y, Tsujimoto A, Fisher NG, Baruth AG, Barkmeier WW, Takamizawa T et al. (2019) The effect of reduced application time of universal adhesives on enamel bond fatigue durability and surface morphology. Oper Dent 44, 42-53.

5. Tsujimoto A, Shimatani Y, Nojiri K, Barkmieier WW, Markham MD, Takamizawa T et al. (2019) Influence of surface wetness on bonding effectiveness of universal adhesives in etch-and-rinse mode. Eur J Oral Sci 127, 162-169.

6. Tsujimoto A, Barkmeier WW, Hosoya Y, Nojiri K, Nagura Y, Takamizawa T et al. (2017) Comparison of bond fatigue durability to enamel of universal adhesives and two-step selfetch adhesives in self-etch mode. Am J Dent 30, 279-284.

7. Suda S, Tsujimoto A, Barkmeier WW, Nojiri K, Nagura Y, Takamizawa T et al. (2018) Comparison of enamel bond fatigue durability between universal adhesives and two-step self-etch adhesives: effect of phosphoric acid pre-etching. Dent Mater J 37, 244-255.

8. Tsujimoto A, Barkmeier WW, Takamizawa T, Watanabe H, Johnson WW, Latta MA et al. (2017) Comparison between universal adhesives and two-step self-etch adhesives in terms of dentin bond fatigue durability in self-etch mode. Eur J Oral Sci 125, 215-222.

9. Irmak Ö, Yaman BC, Orhan EO, Ozer F, Blatz MB (2018) Effect of rubbing force magnitude on bond strength of universal adhesives applied in self-etch mode. Dent Mater J 37, 139-145.

10. Erickson RL, De Gee AJ, Feilzer AJ (2006) Fatigue testing of enamel bonds with self-etch and total-etch adhesive systems. Dent Mater 22, 981-987.

11. Erickson RL, De Gee AJ, Feilzer AJ (2008) Effect of pre-etching enamel on fatigue of self-etch adhesive bonds. Dent Mater 24, 117-123.

12. Barkmeier WW, Erickson RL, Latta MA (2009) Fatigue limits of enamel bonds with moist and dry techniques. Dent Mater 25, 1527-1531.

13. Takamizawa T, Scheidel DD, Barkmeier WW, Erickson RL, Tsujimoto A, Latta MA et al. (2016) Influence of frequency on shear fatigue strength of resin composite to enamel bonds using self-etch adhesives. J Mech Behav Biomed Mater 62, 291-298.

14. Tsujimoto A, Barkmeier WW, Erickson RL, Takamizawa T, Latta MA, Miyazaki M (2018) Influence of the number of cycles on shear fatigue strength of resin composite bonded to enamel and dentin using dental adhesives in self-etching mode. Dent Mater J 37, 113-121.

15. Tsujimoto A, Barkmeier WW, Erickson RL, Fischer NG, Markham MD, Takamizawa T et al. (2018) Shear fatigue strength of resin bonded to dentin under cyclic fatigue at physiological frequency. Eur J Oral Sci 126, 316-325.

16. Tian F, Zhou L, Zhang Z, Niu L, Zhang L, Chen C et al. (2016) Paucity of nanolayering in resin-dentin interfaces of MDP-based adhesives. J Dent Res 95, 380-387.

17. Inoue N, Tsujimoto A, Takimoto M, Otsuka E, Endo H, Takamizawa T et al. (2010) Surface free-energy measurements as indicators of the bonding characteristics of single-step self-etching adhesives. Eur J Oral Sci 118, 525-530.

18. Nagura Y, Tsujimoto A, Barkmeier WW, Watanabe H, Johnson WW, Takamizawa T el al. (2018) Relationship between bond fatigue durability and surface free energy characteristics of universal adhesives to enamel. Eur J Oral Sci 126, 135-145.

19. Van Noort R, Noroozi S, Howard IC, Cardew G (1989) A critique of bond strength measurements. J Dent 17, 61-67.

20. Jin XZ, Homaei E, Matinlinna JP, Tsoi JK (2016) A new concept and finite-element study on dental bond strength tests. Dent Mater 32, e238-e250.

21. Cheetham JJ, Palamara JE, Tyas MJ, Burrow MF (2013) A comparison of the micro-shear bond strength and failure mode of non-enclosed and mold-enclosed luting cements bonded to metal. Dent Mater J 32, 896-905.

22. Cheetham JJ, Palamara JE, Tyas MJ, Burrow MF (2014) A comparison of the shear bond strength and failure mode to metals of unsupported and supported luting cement specimens. J Adhes Dent 16, 251-260.

23. Rosa WL, Piva E, Silva AF (2015) Bond strength of universal adhesives: a systematic review and meta-analysis. J Dent $43,765-776$

24. Tsujimoto A, Iwasa M, Shimamura Y, Murayama R, Takamizawa T, Miyazaki M (2010) Enamel bonding of single-step self-etch adhesives: influence of surface free energy characteristics. J Dent 38, 123-130.

25. Tay FR, Pashley DH, Suh BI, Carvalho RM, Itthagarun A (2002) Single-step adhesives are permeable membranes. J Dent 30, 371-382

26. Tay FR, Pashley DH, Garcia-Godoy F, Yiu CK (2004) Single-step, self-etch adhesives behave as permeable membranes after polymerization. Part II. Silver tracer penetration evidence. Am J Dent 17, 315-322.

27. Yoshioka M, Yoshida Y, Inoue S, Lambrechts P, Vanherle G, Nomura Y et al. (2002) Adhesion/decalcification mechanisms of acid interactions with human hard tissues. J Biomed Mater Res 59, 56-62.

28. Van Landuyt KL, Yoshida Y, Hirata I, Snauwaert J, De Munck J, Okazaki M et al. (2008) Influence of the chemical structure of functional monomers on their adhesive performance. J Dent Res 87, 757-761.

29. Sugimura R, Tsujimoto A, Hosoya Y, Fischer NG, Barkmeier WW, Takamizawa T et al. (2019) Surface moisture influence on etch-and-rinse universal adhesive bonding. Am J Dent 32, 33-38.

30. Nagakane K, Yoshida Y, HirataI I, Fukuda R, Nakayama Y, Shirai K et al. (2006) Analysis of chemical interaction of 4-MET with hydroxyapatite using XPS. Dent Mater J 25, 645649.

31. Yoshida Y, Nagakane K, Fukuda R, Nakayama Y, Okazaki M, Shintani H et al. (2004) Comparative study on adhesive performance of functional monomers. J Dent Res 83, 454458. 